\title{
Anatomical location of the vertebrobasilar junction: computed tomography morphometrics for planning endoscopic transsphenoidal transclival approaches
}

\author{
A. Er, M. Bozdag \\ Department of Radiology, Tepecik Training and Research Hospital, Izmir, Turkey
}

[Received: 13 February 2020; Accepted: 12 March 2020]

Background: The aim of this study was to determine the anatomical localisation and morphometry of vertebrobasilar junction (VBJ) by computed tomography (CT) images which may be helpful in planning the correct procedure before surgery such as endoscopic transsphenoidal transclival approach to the retroclival space. Materials and methods: Vertebrobasilar junction level was determined on axial, coronal reformat and sagittal reformat images. Clivus length, the distances of the $V B J$ to the upper and lower end of the clivus and to the bottom of the sphenoid sinus were measured. In addition, the position and distance of the VBJ relative to the midline were measured. The vertebral artery dominance was determined and the position of VBJ relative to the midline was evaluated.

Results: When compared by gender, 1, a, b and c values were significantly longer in males than in females $(p<0.05)$. The location of the bottom of the sphenoid sinus was higher than the VBJ level in 263 (98.1\%) cases, equal to the VBJ level in $1(0.4 \%)$ case, and lower than the VBJ level in $4(1.5 \%)$ cases. There was no statistically significant difference between the distances to the midline when the $V B J s$ with right and left localisation were compared $(p>0.05)$. A statistically significant relationship was found between vertebral artery predominance and localisation of VBJ relative to the midline $(p<0.001)$.

Conclusions: Careful perusal of CT images and the described VBJ morphometrics can help in accurate procedure planning to avoid basilar artery injury. (Folia Morphol 2020; 79, 3: 445-449)

Key words: vertebro-basilar junction, clivus, endoscopic transsphenoidal transclival approach, computed tomography

\section{INTRODUCTION}

The posterior cranial fossa, including the anterior surface of the clivus and the brainstem, is considered the most difficult region to access in skull base surgery. Despite the rapid development of various surgical techniques in recent years, surgical inter- ventions in this area remain challenging due to the complex anatomical environment involving the main blood vessels and cranial nerves. Endoscopic transsphenoidal transclival approach provides the ability to overcome some of the above mentioned limiting factors and achieve successful surgical intervention

Address for correspondence: A. Er, MD, Department of Radiology, Tepecik Training and Research Hospital, 1140/1 street, No: 1 Yenisehir - Konak, Izmir, Turkey, tel: +90 23246969 69, fax: +90 23243307 56, e-mail: alier1717@yahoo.com 
in the posterior cranial fossa anterior region structures $[3,11]$.

Knowing the anatomical localisation and morphometry of the vertebrobasilar junction (VBJ), an important anatomical structure in the skull base, can help prevent any damage that may occur during surgery. The aim of this study was to determine the anatomical localisation and morphometry of VBJ by computed tomography (CT) images which may be helpful in planning the correct procedure before surgery.

\section{MATERIALS AND METHODS}

Computed tomography angiography images of 268 patients who underwent multi-detector cerebral CT angiography in our institution between January 2014 and January 2019 were reviewed retrospectively to determine the anatomical location of the VBJ. The patients underwent cerebral $C T$ angiography for many different possible conditions such as cerebral aneurysm, intracerebral haemorrhage, and vascular malformation. All cases with a history of surgical or interventional treatment, cerebral obstructive disease, variations of vertebral artery (VA) (e.g., basilar fenestration, vertebral artery terminating in the posterior inferior cerebellar artery, etc.) and with poor image quality due to patient movement or technical problems were excluded. Approval for this retrospective study was obtained from the review board of Tepecik Training and Research Hospital.

Computed tomography angiography examinations were performed using a 128-slice CT scanner (SOMATOM Definition Edge, Siemens Healthcare, Erlangen, Germany). After pre-contrast images, a total of $80-90 \mathrm{~mL}$ of contrast medium with a high iodine concentration $(370-400 \mathrm{mg} / \mathrm{mL}$ ) was injected with a flow rate of $5 \mathrm{~mL} / \mathrm{s}$. Scan parameters are as follows: $120 \mathrm{kV}$; $225 \mathrm{~mA}$; section thickness: $0.5 \mathrm{~mm}$; reconstruction range: $0.3 \mathrm{~mm}$. CT angiography data were obtained in the caudocranial direction from the aortic arch to the vertex level in a supine position. In the course of the study readings, all data were transferred from the workstation (Aquarius workstation, TeraRecon, San Mateo, California, USA) via internal network connections, providing multiplanar image reformatting (MPR) images. All measurements were performed by a single radiologist.

The measurement parameters we have determined for the anatomical localisation and morphometry of VBJ are described and listed in the following paragraphs:
First, coronal and sagittal reformat images were created from axial source images. Then, the midlines of the falx cerebri and odontoid process on axial and coronal MPR images were taken as reference and the midline was determined accordingly. Then, the level of VBJ was determined in axial, coronal reformat and sagittal reformat images (Fig. 1A-C).

Secondly, in the series with the midline line determined on axial and coronal MPR images, two lines were drawn from the upper (2) and lower (4) ends of the clivus along the long axis of the clivus (1) perpendicular to the line drawn from the midline (clivus length) in the sagittal MPR image. The distance between the upper end of the clivus and the VBJ (a) and the distance between the lower end of the clivus and the VBJ (b) (Fig. 1A-C) were then measured.

Thirdly, the lowest point of the sphenoid sinus (SS) in the sagittal image was determined, and another line was drawn perpendicular to the line drawn from the mid-section along the long axis of the clivus (3). The distance between this line and VBJ (c) was measured (Fig. 1A-C).

Fourth, the position of VBJ relative to the midline was determined on axial and coronal MPR images. If the determined VBJ position was located on the right or left of the midline, its distance from the midline was recorded on coronal reformatted images (d). In addition, axial and coronal MPR images revealed which VA was dominant (Fig. 2A-C).

\section{Statistical analysis}

Statistical analysis was performed using computer software (Statistical Package for Social Sciences version 22.0, SPSS Inc., Chicago, Illinois, USA). Discrete and continuous data were represented as numbers (n) and percentages (\%) for qualitative (categorical) variables and means \pm standard deviation (SD) for continuous variables. The normality of distribution of the parameters was assessed using the Shapiro-Wilk test. The distance from the upper point of clivus to VBJ, the distance from the lower point of clivus to $V B J$, clivus length (from the upper to the lower point of clivus), the distance from the lowest point of the sphenoid sinus to the VBJ and the difference of distances of VBJ to midline among genders were evaluated with independent-sample t-test for normally distributed variables and with Mann-Whitney $U$ test for non-normally distributed variables. Chi-square test was recruited to assess any relationship between gender, anatomic location of VBJ, and the dominant 


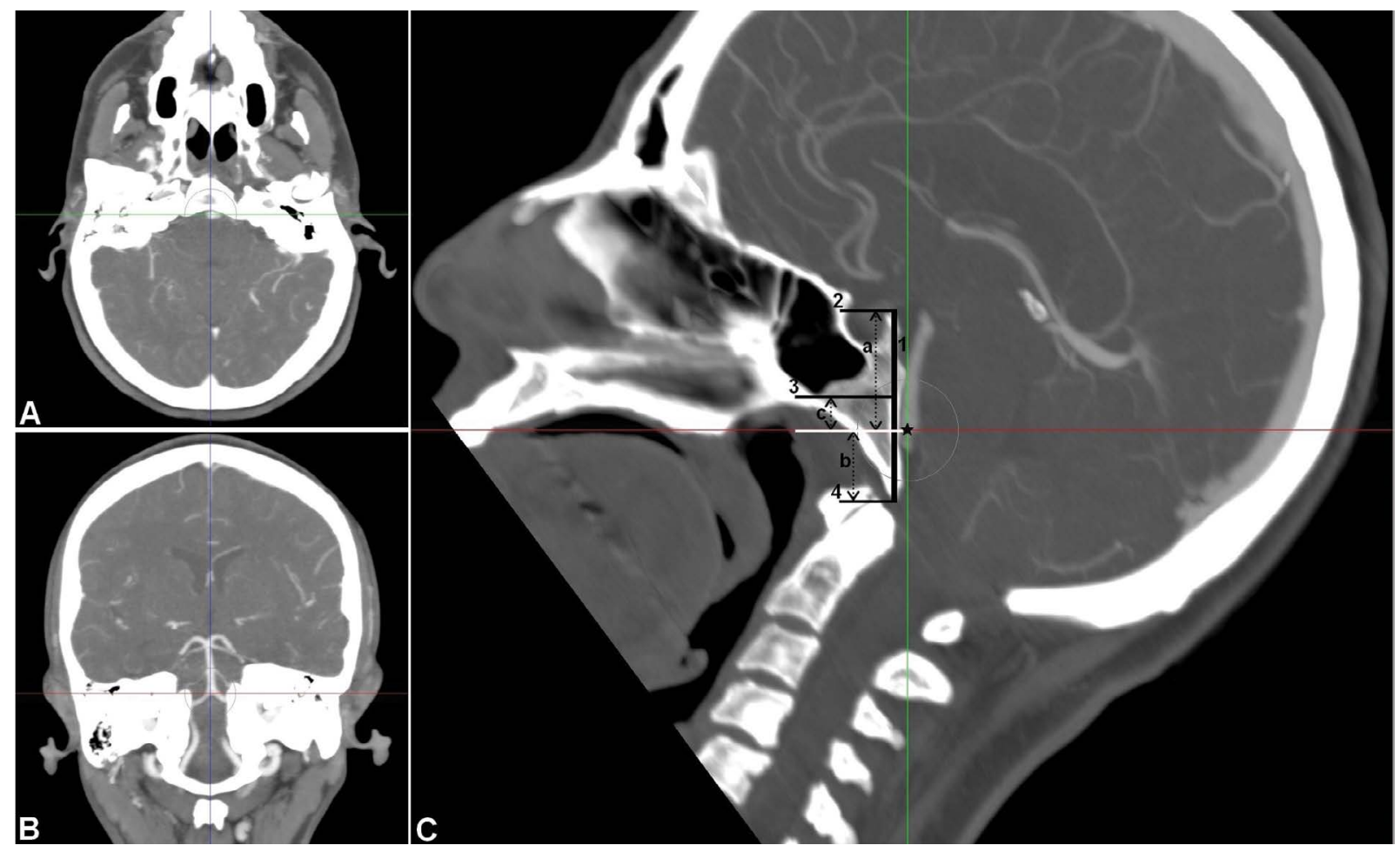

Figure 1. Multiplanar reconstruction (MPR) images; A. Axial image; B. Coronal view; C. Sagittal view of vertebrobasilar junction (VBJ) (star). The intersection of the red, blue and red lines in the three plans shows VBJ. The line drawn from the middle section along the long axis of the clivus (1). The line perpendicular drawn from the upper end of the clivus (2). The line perpendicular drawn from the lower end of the clivus (4). The line drawn perpendicular from the bottom most point of the sphenoid sinus to the clivus (3). Distance between the upper end of clivus and level of VBJ (white line) (a). Distance between the lower end of clivus and level of VBJ (white line) (b). The distance between the bottom most point of the sphenoid sinus and the level of VBJ (white line) (c).

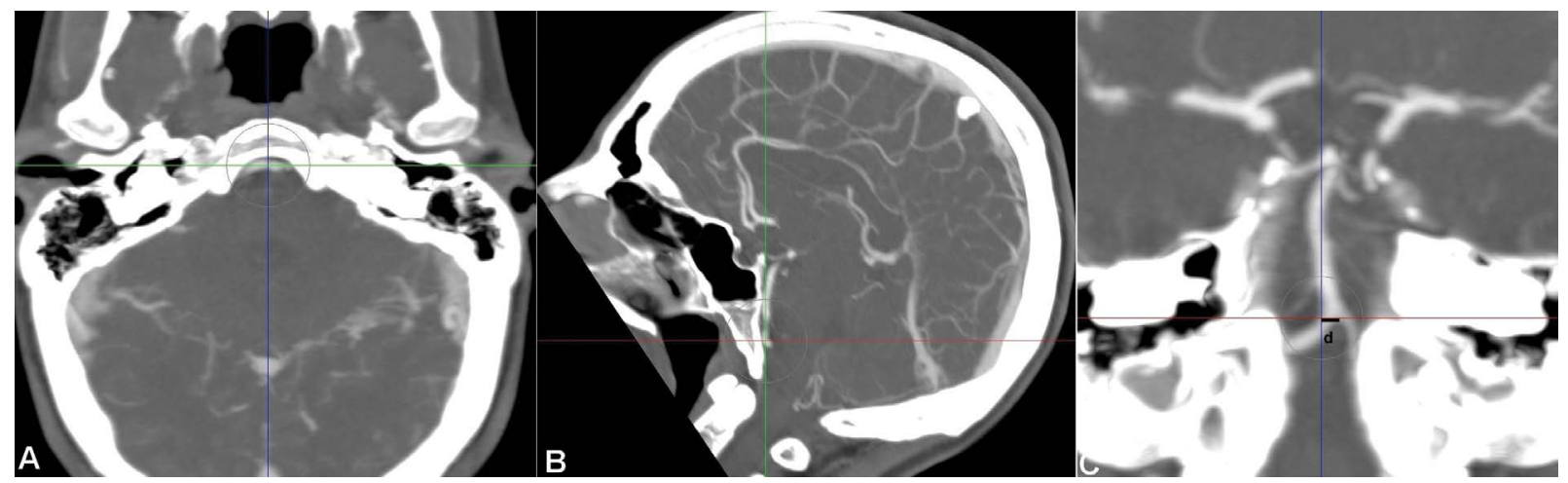

Figure 2. Multiplanar reconstruction images of vertebrobasilar junction (VBJ); A. Axial image; B. Sagittal view; C. Coronal view; $d-$ the distance between the midline and the VBJ.

VA side. $P$ values less than 0,05 was considered statistically significant.

\section{RESULTS}

A total of 268 patients were included in the study. Of those, 144 were male $(53.7 \%), 124$ were female $(46.3 \%)$, and the mean age was 45.8 years (range 18-68 years). In males, the 1 , a, b, c values were sig- nificantly longer than those in females $(p<0.05)$. The measurements of $1, a, b$ and $c$ are presented in Table 1 .

The location of the bottom most point of the sphenoid sinus relative to the VBJ level was found to be above in $263(98.1 \%)$ cases, equal in $1(0.4 \%)$ case and lower in $4(1.5 \%)$ cases.

Of the VBJs, 76 (28.4\%) were located in the midline, $79(29.5 \%)$ were located on the right side of the 
Table 1. Morphometry results

\begin{tabular}{cccc}
\hline Item & \multicolumn{2}{c}{ Mean diameter $[\mathrm{mm}] \pm$ standard deviation } & P variances \\
\cline { 2 - 3 } & Woman & Men & \\
\hline 1 & $44.4 \pm 3.6$ & $48.8 \pm 4.1$ & $<0.05$ \\
a & $28.2 \pm 4.2$ & $30.7 \pm 4.4$ & $<0.05$ \\
b & $16.4 \pm 3.8$ & $18.2 \pm 4.1$ & $<0.05$ \\
c & $12.1 \pm 6.0$ & $13.1 \pm 5.1$ & $<0.05$ \\
\hline
\end{tabular}

1 - clivus length; $a-$ the distance between the upper end of the clivus and the vertebrobasilar junction (VBJ); $b$ - the distance between the lower end of the clivus and the VBJ; $c$ - the distance between lowest point of the sphenoid sinus and VBJ

midline and 113 (42.2\%) were located on the left side. The distance to the midline (d) was $4.1 \pm 2.4 \mathrm{~mm}$ in cases where VBJ was located on the left and $3.8 \pm$ $\pm 2.1 \mathrm{~mm}$ in cases where it was located on the right. No statistically significant difference was found between the cases with VBJs located on the right and on the left, in terms of distance to the midline ( $p>0.05)$. No significant relationship was found between the relative placement of VBJ to the midline and gender $(p>0.05)$.

When VA dominance was evaluated, 145 (54.1\%) had no dominance, 38 (14.2\%) had right and 85 (31.7\%) had left dominance. There was no significant difference between the sexes in terms of VA dominance ( $p>0.05)$.

When the relationship between the predominance of vertebral arteries and the localisation of VBJ relative to the midline was evaluated, the following results were observed: In 6 (15.8\%) of the patients with right VA predominance the VBJ was located in the midline, in $1(2.6 \%)$ patient it was located on the right side of the midline, and in 31 (81.6\%) it was located on the left side. There was a statistically significant relationship between right VA dominance and localisation of $V B J$ relative to the midline ( $p<0.001, \chi^{2}$ test). In $13(15.3 \%)$ patients with left VA dominance, the VBJ was located in the midline, in $19(22.3 \%)$ to the left of the midline and in $53(62.4 \%)$ to the right of the midline. There was also a statistically significant relationship between the predominance of the left VA and the localisation of VBJ relative to the midline ( $p<0.001, \chi^{2}$ test).

\section{DISCUSSION}

The endoscopic transsphenoidal transclival approach is an important corridor for midline interventions of the anterior section of the posterior fossa and skull base surgeries. In order to avoid possible complications, knowing the anatomical localisation and morphometry of VBJ is important in clinical practice. Clivus is divided into three surgically relevant anatomic segments: upper, middle and lower by referring to the dorsum sellae, Dorello's canal and pars nervosa of the jugular foramen. The upper section approach is rarely used in operations. The middle section is used for access to the anterior of the pons, prepontine cistern, basilar truncus, and the cisternal segment of the sixth cranial nerve. The lower section, on the other hand, is used for access to premedullary cistern, VBJ, vertebral arteries, posterior inferior cerebellar arteries and cisternal segments of IX-XII cranial nerves $[3,5,11]$.

The transsphenoidal corridor is a convenient way to access the mid-clival and anterior part lesions of the posterior fossa. In addition to lesion localisation, origin, and related peripheral structures, pneumatisation of the sphenoidal sinus is important in planning a surgical approach and the presence of a well-pneumatised sphenoid sinus is quite beneficial $[5,11]$. However, the length, height, and width of the sphenoid sinus increase with age and vary from person to person, and many different pneumatisation patterns have been described in the sagittal and coronal planes [9]. As can be seen here, there is no standardised view acceptable for every patient of the sphenoid sinus. Despite these non-standardised appearances in the sphenoid sinus, which appeared to be a challenge in preoperative planning, the bottom most point of the sphenoid sinus was located above the VBJ level in a very high $98.1 \%$ of the cases in our study. This data that we obtained and the average distances between these anatomical structures can well be considered as a standardisation point.

Vertebral artery is divided into four segments during its course and the fourth segment, known as the intracranial segment, starts from the foramen magnum level, merges with the corresponding segment at the pontomedullary level and forms VBJ. Although the anatomy of the structure is well known, especially starting with VA $4^{\text {th }}$ segment and ending with VBJ, it is important to have information about the anatomical variability of these structures, especially in terms of planning for brain surgery, head and face surgery, and vascular surgery $[4,6]$. Songur et al. [10] described the position of VBJ associated with the bulbopontin sulcus and found that $20 \%$ of VBJ was at this level, $67 \%$ was below this level, and $12 \%$ was above this level. In our study, we selected the upper and lower ends of the clivus as reference points in order to standardise VBJ 
localisation and generate numerical data in operation planning. We think that the distances between the upper and lower extremities of VBJ in male and female patients differ significantly and the morphometric data obtained from both genders are important.

Akkasoglu et al. [1] reported that there may be a connection between VA dominancy and basilar artery morphometry. Zhu et al. [12] reported that the predominance of the left VA was more common than the right (56.4\%). Cloud and Markus [2] stated that the prevalence of left VA predominance was $50 \%$ and in $25 \%$ cases there was no dominance. According to our findings, a statistically significant relationship was found between the VA dominancy and the localisation of VBJ relative to the midline, and we found that VBJ was predominantly located to the left of the midline in the presence of the right VA dominant artery, and VBJ was predominantly to the right of the midline in the presence of the left VA dominant artery. In our series, $54 \%$ of patients did not have dominance between both VA, followed by left VA dominance. Right VA dominance was the rarest. We think that these findings may be helpful and guiding in preoperative planning.

In the endoscopic transclival approach, the extra-intradural space from the crista galli to the craniovertebral junction is broadly visualised compared to the transcranial method, resulting in a good surgical corridor which is an advantage. Also, there is no need to perform traction on various structures of the brain during this approach $[7,8]$. For these reasons, the endoscopic transsphenoidal transclival approach is increasingly preferred in the management of brain anterior lesions and the importance of detailed recognition of anatomical structures in this localisation is increasing.

\section{CONCLUSIONS}

The data obtained by analysing the anatomical localisation and morphometry of VBJ will help to plan the surgical approach for the lesions of the clivus and the anterior part of the posterior fossa and to prevent the associated complications.

\section{REFERENCES}

1. Akkasoglu S, Aldur M, Sargon M, et al. Morphometry and geometry of the formation of basilar artery.
Med-Science. 2019; 8(4): 980-985, doi: 10.5455/medscience.2019.08.9076.

2. Cloud GC, Markus HS. Diagnosis and management of vertebral artery stenosis. Ojm-Int J Med (OJM). 2013; 96: 27-34

3. de Notaris M, Cavallo LM, Prats-Galino A, et al. Endoscopic endonasal transclival approach and retrosigmoid approach to the clival and petroclival regions. Neurosurgery. 2009; 65(6 Suppl): 42-50; discussion 50, doi: 10.1227/01.NEU.0000347001.62158.57, indexed in Pubmed: 19935001.

4. Dzierżanowski J, Szarmach A, Baścik B, et al. Intracranial region of the vertebral artery: morphometric study in the context of clinical usefulness. Folia Morphol. 2017; 76(3): 379-387, doi: 10.5603/FM.a2017.0021, indexed in Pubmed: 28281722.

5. Cossu G, Daniel RT, George M, et al. New frontiers in managing clival tumors - the extended endoscopic endonasal approach. Endoscopy - Innovative Uses and Emerging Technologies. 2015, doi: 10.5772/60553.

6. Gupta T. Quantitative anatomy of vertebral artery groove on the posterior arch of atlas in relation to spinal surgical procedures. Surg Radiol Anat. 2008; 30(3): 239-242, doi: 10.1007/s00276-008-0313-x, indexed in Pubmed: 18253689 .

7. Sanmillan JL, Lawton MT, Rincon-Torroella J, et al. Assessment of the endoscopic endonasal transclival approach for surgical clipping of anterior pontine anterior-inferior cerebellar artery aneurysms. World Neurosurg. 2016; 89: 368-375, doi: 10.1016/j.wneu.2016.01.081, indexed in Pubmed: 26852706.

8. Shkarubo A, Koval K, Chernov I, et al. Endoscopic endonasal transclival removal of tumors of the clivus and anterior region of the posterior cranial fossa (results of surgical treatment of 140 patients). Chinese Neurosurg J. 2018; 4(1), doi: 10.1186/s41016-018-0144-5.

9. Singh H, Greenfield JP, Anand VK, Schwartz TH. Pediatric Endonasal Endoscopic Skull Base Surgery: A case-Based Manuel. Endo Press, Tuttlingen 2016.

10. Songur A, Gonul Y, Ozen OA, et al. Variations in the intracranial vertebrobasilar system. Surg Radiol Anat. 2008; 30(3): 257-264, doi: 10.1007/s00276-008-0309-6, indexed in Pubmed: 18253692.

11. Ting $E$, Ting $F$, Chia $G$, et al. Imaging in endoscopic endonasal skull base surgery. In: Chong V(ed.). Skull Base Imaging. Elsevier. Skull Base Imaging. 2018: 65-82, doi: 10.1016/b978-0-323-48563-0.00004-0.

12. Zhu W, Wang YF, Dong XF, et al. Study on the correlation of vertebral artery dominance, basilar artery curvature and posterior circulation infarction. Acta Neurol Belg. 2016; 116(3): 287-293, doi: 10.1007/s13760-015-0570-5, indexed in Pubmed: 26615535. 